\title{
An Extended Theory of General Relativity Unifying the Matter-Antimatter States
}

\author{
Patrick Marquet \\ Former Head of Research, Laboratory of Applied Astronomy Physics \\ Observatoire de Paris, 75014 Paris, France \\ Private: 7, rue du 11Novembre, 94350 Villiers-Paris, France \\ Tel: 33-149-303-342Ｅ-mail: patrick.marquet6@wanadoo.fr
}

Received: November 15, 2010 Accepted: November 30, 2010 doi:10.5539/apr.v3n1p60

\begin{abstract}
This paper follows one of our earlier publications detailing an extended theory of General Relativity (GR) with two types of curvatures.

In this framework, we first extend the Riemannian spinor theory in the formalism of A.Lichnerowicz.

We then suggest that the non vanishing covariant derivative of the metric tensor linked to the extra curvature of our extended GR theory, be related to the trace of a specific Hermitean matrix.

In the Riemannian theory, this matrix reduces to the classical gamma matrix that is precisely designed to distinguish the spin one half fields from its anti Fermionic counterpart.

Our extended General Relativity theory therefore appears as unifying the Fermion-anti Fermion field states, and would legitimate the hidden medium concept introduced by Louis de Broglie in his attempt to explain the matter-antimatter symmetry.
\end{abstract}

Keywords: EGR theory, Covariant 1-spinor, Contravariant 1-spinor

\section{Basics of EGR Theory}

\subsection{General}

We recall here the main results of the Extended Theory of General Relativity (EGR theory) which is published in [Marquet. P, 2009].

Unlikein the Riemannian theory, we consider a four dimensional manifold $\mathfrak{T}$ endowed with two types of curvatures implying a semi-affine connection.

(space-time latin indices run here from $a=b=1,2,3,4$ )

On the manifold $\mathscr{N}$ referred to a natural basis $\left\{e_{a}\right\}$, it is known that the connection coefficients $\Gamma_{a b}^{c}$ can be generally decomposed as follows

$$
\Gamma_{a b}^{c}=\left\{\begin{array}{c}
c \\
a b
\end{array}\right\}+K_{a b}^{c}+\left(\Gamma_{a b}^{c}\right) \mathbf{s}
$$

$\left\{\begin{array}{l}c \\ a b\end{array}\right\}$ are the Christoffel symbols of the second kind.

$K_{a b}^{c}$ is the contorsion tensor which includes the torsion tensor $T_{b c}^{a}=1 / 2\left[\Gamma^{a}{ }_{[b c]}-\Gamma^{a}{ }_{[c b]}\right]$.

$\left(\Gamma_{a b) \mathrm{S}}^{c}=1 / 2 g^{c e}\left(\mathrm{D}_{b} g_{a e}+\mathrm{D}_{a} g_{b e}-\mathrm{D}_{e} g_{a b}\right)\right.$ is the segmental connection formed with the general covariant derivatives (denoted by $\mathrm{D})$, of the metric tensor:

$$
\mathrm{D}_{c} g_{a b}=\partial_{c} g_{a b}-\Gamma_{a c, b}-\Gamma_{b c, a} .
$$

This latter connection characterizes a particular property of the manifold $\Re$ related to a specific type of curvature, known as the segmental curvature.

In a dual basis $\theta^{a}$ defined on $\Re$, to any parallel transported vector along a closed path, can be then associated the following forms

- A rotation curvature $\Omega_{b}^{a}=-1 / 2 R^{a}{ }_{b c d} \theta^{c} \Lambda \theta^{d}$ 
- A torsion $\quad \Omega^{a}=1 / 2 T^{a}{ }_{c d} \theta^{c} \Lambda \theta^{d}$

- A segmental curvature $\Omega=-1 / 2 R^{a}{ }_{a c d} \theta^{c} \Lambda \theta^{d}$

We postulate that the manifold $\Re$ is torsion free with 40 general symmetric connection coefficients

$$
\Gamma_{b c}^{a}=\{a b c\}+\left(\Gamma_{b c}^{a}\right)_{S}
$$

The components $R^{e}{ }_{a c d}$ of the general curvature tensor are assumed to have the same form as the ones of the Riemann tensor

$$
R_{a c d}^{e}=\partial_{d} \Gamma_{a c}^{e}-\partial_{c} \Gamma_{a d}^{e}+\Gamma_{a c}^{e} \Gamma_{f d}^{f}-\Gamma_{a d}^{f} \Gamma_{f c}^{e}
$$

\subsection{The modified action}

\subsubsection{Eulerian equations}

From the components of the Ricci like tensor formed with the global $\Gamma_{b c}^{a}$

$$
R_{b c}=\partial_{a} \Gamma_{b c}^{a}-\partial_{c} \Gamma_{b a}^{a}+\Gamma_{b c}^{d} \Gamma_{d a}^{a}-\Gamma_{b a}^{d} \Gamma_{d c}^{a}
$$

we define the tensor density

$$
\mathfrak{R}^{a b}=R^{a b}(-g)^{1 / 2}
$$

from which we build the invariant density

$$
\mathscr{H}=\mathfrak{R}^{a b} R_{a b}
$$

with $\mathscr{R}^{a b}=\partial \mathscr{H} / \partial R_{a b}$

Following a suggestion by Einstein, we consider the connection coefficients (1.0) as the true field variables, so that for a variation $\delta \Gamma^{a}{ }_{b c}$, the action principle

$$
\delta \S=\int \delta \mathscr{H} d^{4} x=0
$$

be written

$$
\begin{gathered}
\delta \mathcal{S}=\int\left[\left(\partial \mathcal{H C} / \partial \Gamma_{b c}^{a}\right) \delta \Gamma_{b c}^{a}+\left(\partial \mathcal{H} / \partial\left(\partial_{e} \Gamma_{b c}^{a}\right)\right) \cdot \delta\left(\partial_{e} \Gamma^{a}{ }_{b c}\right)\right] d^{4} x=0 \\
=\int\left[\left(\mathcal{R}^{b c} \partial R_{b c} \delta \Gamma^{a}{ }_{d e}\right) / \partial \Gamma^{a}{ }_{d e}+\left(\mathfrak{R}^{b c} \partial R_{b c} \delta\left(\partial_{f} \Gamma^{a}{ }_{d e}\right)\right) \partial / \partial\left(\partial_{f} \Gamma_{d e}{ }_{d e}\right)\right] d^{4} x=0
\end{gathered}
$$

and integrating by parts, we obtain

$$
\delta\left[\left[\left(\Re^{b c} \partial R_{b c} / \partial \Gamma_{d e}^{a}\right)-\partial_{f}\left(\left(\Re^{b c} \partial R_{b c}\right) / \partial\left(\partial_{f} \Gamma_{d e}^{a}\right)\right)\right] \delta \Gamma_{d e}^{a}+\int_{f}\left[\Re^{b c} \partial R_{b c} \delta \Gamma_{d e}^{a} / \partial\left(\partial_{f} \Gamma^{a}{ }_{d e}\right)\right] d^{4} x=0\right.
$$

If the variations $\Gamma_{d e}^{a}$ are zero on the integration boundary, the last divergence integral does not contribute, so the condition (1.5) reduces to

$$
\delta \int \mathcal{R}^{a b} R_{a b} d{ }^{4} x=\int\left[Q_{a}^{b c} \delta\left(\Gamma^{a}{ }_{b c}\right)\right] d{ }^{4} x=0
$$

with

$$
Q_{a}^{b c}=\mathfrak{R}^{d e} \partial R_{d e} / \partial \Gamma_{b c}^{a}-\partial_{f}\left[\mathcal{R}^{d e} \partial R_{d e} / \partial\left(\partial_{f} \Gamma_{b c}^{a}\right)\right]
$$

The stationary principle for symmetrical $\Gamma_{b c}^{a}$, leads to the eulerian equations

$$
Q_{a}^{(b c)}=1 / 2\left(Q_{a}^{b c}+Q_{a}{ }^{c b)}=0\right.
$$

From the expression (1.1), we next calculate the derivatives

$$
\partial R_{d f} / \partial\left(\partial_{e} \Gamma{ }_{b c}^{a}\right)=\delta^{e}{ }_{m} \delta^{b}{ }_{d} \delta^{c}{ }_{f} \delta^{m}{ }_{a}-\delta^{e}{ }_{f} \delta^{b}{ }_{d} \delta^{c}{ }_{m} \delta^{m}{ }_{a}
$$

and

$$
\partial R_{d f} / \partial \Gamma_{b c}^{a}=\delta^{l}{ }_{m} \delta^{b}{ }_{d} \delta^{c}{ }_{f} \Gamma^{m}{ }_{l m}+\delta^{m}{ }_{a} \delta^{b}{ }_{l} \delta^{c}{ }_{f} \Gamma^{l}{ }_{d m}-\delta^{l}{ }_{a} \delta^{b}{ }_{d} \delta^{c}{ }_{f} \Gamma^{m}{ }_{l f}-\delta^{m}{ }_{a} \delta^{b}{ }_{l} \delta^{c}{ }_{f} \Gamma^{l}{ }_{d m}
$$

substituting now into (1.7)

$$
\begin{gathered}
Q_{a}^{b c}=\partial_{a} \mathfrak{R}^{b c}-\delta_{a}^{c} \partial_{e} \mathfrak{R}^{b e}-\mathfrak{R}^{b c} \Gamma^{m}{ }_{a m}-\delta_{a}^{f} \mathfrak{R}^{d c} \Gamma_{d f}^{b}+\mathfrak{R}^{b f} \Gamma^{c}{ }_{a f}+\mathfrak{R}^{f c} \Gamma_{f a}^{b} \\
=\mathfrak{R}^{b c}{ }_{a}{ }_{a}-\delta^{c}{ }_{a} \mathfrak{R}^{b e}{ }_{,}{ }_{e}
\end{gathered}
$$

with

$$
\mathfrak{R}^{b c}{ }_{, a}=\partial_{a} \mathfrak{R}^{b c}+\Gamma_{e a}^{b} \mathfrak{R}^{e c}+\Gamma_{e a}^{c} \mathfrak{R}^{e b}-\Gamma_{a e}^{e} \mathfrak{R}^{b c}
$$

(the ', or $\mathrm{D}$ are the covariant derivatives constructed with the global connection $\Gamma$ defined in (1.0))

The condition (1.8) explicitly yields

$$
\left(R^{b c}+\mathscr{R}^{c b)}{ }_{{ }_{a}}{ }_{a}-\delta^{c}{ }_{a} \mathfrak{R}^{b e}{ }_{{ }_{\mathrm{e}}}-\delta^{b}{ }_{a} \mathfrak{R}^{c e,}{ }_{{ }_{e}}=0\right.
$$




\subsection{Connection coefficients}

In order to determine the exact form of the connection, we first decompose $\mathfrak{R}^{b c}$ into the metric tensor density

$$
\mathcal{G}^{b c}=(-g)^{1 / 2} g^{b c}
$$

and two further parts $\mathcal{E}^{b c}+\mathfrak{Q}^{b c},\left(\mathfrak{Q}^{b c}\right.$ being antisymmetric):

$$
\mathfrak{R}^{b c}=\left(\mathcal{G}^{b c}+\mathcal{E}^{b c}\right)+\mathfrak{Q}^{b c}
$$

the quantity between brackets represents the Riemann Ricci tensor density

$$
R^{b c}(-g)^{1 / 2}=\mathfrak{R}^{b c}=\left(\mathcal{G}^{b c}+\mathcal{E}^{b c}\right)
$$

so that when $\mathbb{Q}^{b c}=0,(1.13)$ reduces as it should, to (1.13)bis.

Consistency of our theory thus leads to impose the following constraint

$$
\left(\mathcal{E}^{b c}\right)^{\prime},{ }_{b}=0
$$

we next set

$$
\mathfrak{g}^{b}=\left(\mathfrak{Q}^{b a}\right)^{\prime},{ }_{a}
$$

with

$$
\mathscr{g}^{a}=(-g)^{1 / 2} J^{a}
$$

Let us now check whether the condition $\mathcal{G}^{b c},{ }_{c}=0$ re-instates a Riemann connection whereby the general curvature tensor $R_{a b}$ (1.1) would reduce to the riemann Ricci tensor $R_{a b}$.

By contracting (1.12) on $c$ and $a$, and taking into account (1.13)ter, one finds

$$
\mathcal{S}^{b c}{ }_{, c}=-5 / 3 \mathscr{g}^{b}
$$

If we now insert (1.15) into (1.12), the conditions (1.8) eventually read

Dividing by $(-g)^{1 / 2}$, one obtains

$$
\mathcal{S}^{b c}{ }_{, a}=-1 / 3\left(\delta^{b}{ }_{a} \varrho^{c}+\delta^{c}{ }_{a} \oint^{b}\right)
$$

$$
\begin{gathered}
\partial_{a} g^{b c}+g^{b c} \partial_{a} \ln (g)^{1 / 2}+\Gamma^{b}{ }_{e a} g^{e c}+\Gamma_{e a}^{c} g^{b e}-\Gamma_{e a}^{e} g^{b c} \\
=-1 / 3\left(\delta^{b}{ }_{a} J^{c}+\delta^{c}{ }_{a} J^{b}\right)
\end{gathered}
$$

so, multiplying through by $g_{b c}$, taking account of $g_{b a} g^{c a}=\delta^{c}{ }_{b}, d g=g g^{b c} d g_{b c}=-g g_{b c} d g^{b c}$, we infer

$$
\Gamma_{a e}^{e}=\partial_{a} \ln (g)^{1 / 2}+1 / 3 J_{a}
$$

Substituting this relation into (1.17) and multiplying by $g_{b d} g_{f c}$ (noting that $d g_{e d}=-g_{e c} g_{b d} d g{ }^{b c}$, we eventually find

$$
\partial_{a} g_{b c}-\Gamma_{b a}^{f} g_{f c}-\Gamma_{c a}^{f} g_{b f}=1 / 3\left[J_{c} g_{a b}+J_{b} g_{a c}-J_{a} g_{b c}\right]=\mathrm{D}_{a} g_{b c}
$$

Interchanging $a$ and $b$, then $a$ and $c$, one obtains two more equations of type (1.19), which are here denoted by (1.19)bis and (1.19)ter (virtual equations).

From the linear combination (1.19)bis $+(1.19)$ ter $-(1.19)$, we finally get the explicit form of the global connexion

$$
\Gamma_{a b}^{d}=\left\{{ }_{a b}^{d}\right\}+\left(\Gamma_{a b}^{d}\right)_{J}=\left\{{ }_{a b}^{d}\right\}+1 / 6\left(\delta_{a}^{d} J_{b}+\delta^{d}{ }_{b} J_{a}-3 g_{a b} J^{d}\right)
$$

Our last equation (1.19) shows that when $J_{a}=0, \mathrm{D}_{a} g_{b c}=0$ and $\mathcal{G}^{a b}{ }^{\prime}{ }_{b}=0$

From (1.18) the condition $J_{a}=0$ implies $\left[\Gamma^{b}{ }_{a e}\right]_{J}=0$, so we actually proved that in this case, the generalized curvature tensor $R_{a b}(1.1)$ reduces to the Riemann Ricci tensor $R_{a b}$.

\subsection{The EGR Curvatures}

1.4.1 The EGR fourth rank curvature tensor

From the connection

$$
\Gamma_{a b}^{d}=\left\{\begin{array}{l}
d \\
a b
\end{array}\right\}+\left(\Gamma_{a b}^{d}\right)_{J}=\left\{\begin{array}{c}
d \\
a b
\end{array}\right\}+(1 / 6)\left(\delta_{a}^{d} J_{b}+\delta^{d}{ }_{b} J_{a}-3 g_{a b} J^{d}\right)
$$

the EGR curvature tensor can be inferred as

$$
\left[R_{b c d}^{a}\right]_{\mathrm{EGR}}=R^{a}{ }_{b c d}+\nabla_{d} \Gamma_{b c}^{a}-\nabla_{c} \Gamma_{b d}^{a}+\Gamma_{b c}^{f} \Gamma_{f d}^{a}-\Gamma_{b d}^{f} \Gamma_{f c}^{a}
$$

Inspection shows that the following relations hold 
let us now contract

$$
\begin{gathered}
R_{d a b ; f}^{e}+R_{d f a ; b}^{e}+R_{d b f ; a}^{e}=0 \\
R_{d a b}^{e}+R_{b d a}^{e}+R_{a b d}^{e}=0
\end{gathered}
$$

$$
\left[R_{d a b}^{e}\right]_{\mathrm{EGR}} g_{c e}=\left[R_{c d a b}\right]_{\mathrm{EGR}}
$$

from $\nabla_{a}\left(\Gamma_{d b}^{e}\right)_{J}$, we notice that $g_{c e} \nabla_{a}\left[\left(\Gamma_{b e}^{e}\right)_{J} \delta_{b}^{e} \delta_{d}^{b}\right]=g_{c d} \nabla_{a}\left(\Gamma_{b e}^{e}\right)_{J}$ and the curvature tensor (1.23) now reads

$$
\begin{gathered}
{\left[R_{c d a b}\right]_{\mathrm{EGR}}=R_{c d a b}+g_{c e} \nabla_{b}\left(\Gamma_{d a}^{e}\right)_{J}-1 / 2 g_{c e}\left[\nabla_{a}\left(\Gamma_{d b}^{e}\right)_{J}+\nabla_{d}\left(\Gamma_{a b}^{e}\right)_{J}\right]} \\
+g_{c e}\left[\left(\Gamma_{f b}^{e}\right)_{J}\left(\Gamma^{f}{ }_{d a}\right)_{J}-\left(\Gamma_{f a}^{e}\right)_{J}\left(\Gamma^{f}{ }_{d b}\right)_{J}\right]+g_{c d}\left[\partial_{a}\left(\Gamma_{b e}^{e}\right)_{J}-\partial_{b}\left(\Gamma_{a e}^{e}\right)_{J}\right]
\end{gathered}
$$

with the definition (1.21) we have

1.4.2 EGR second rank tensor $R_{a b}$

$$
\begin{gathered}
\left(\Gamma_{a d}^{d}\right)_{J}=1 / 3 J_{a} \\
\text { and } \partial_{a}\left(\Gamma_{b d}^{d}\right)_{J}-\partial_{b}\left(\Gamma_{a d}^{d}\right)_{J}=(1 / 3) J_{a b} \\
\text { with } J_{a b}=\partial_{a} J_{b}-\partial_{b} J_{a}
\end{gathered}
$$

The relation (1.24) eventually leads to the contracted tensor

$$
\left[R^{d}{ }_{a b d}\right]_{\mathrm{EGR}}=\left[R_{a b}\right]_{\mathrm{EGR}}=R_{a b}+\nabla_{d}\left(\Gamma_{a b}^{d}\right)_{J}-\nabla_{b}\left(\Gamma_{a d}^{d}\right)_{J}+\left(\Gamma_{a b}^{f}\right)_{J}\left(\Gamma_{f d}^{d}\right)_{J}-\left(\Gamma_{a e}^{f}\right)_{J}\left(\Gamma_{f b}^{e}\right)_{J}
$$

we then have once more the splitting

$$
\left[R_{a b}\right]_{\mathrm{EGR}}=\left[R_{(a b)}\right]_{\mathrm{EGR}}+\left[R_{[a b]}\right]_{\mathrm{EGR}}
$$

where

$$
\begin{gathered}
{\left[R_{(a b)}\right]_{\mathrm{EGR}}=R_{a b}+\nabla_{d}\left(\Gamma_{a b}^{d}\right)_{J}-1 / 2\left[\nabla_{b}\left(\Gamma_{a d}^{d}\right)_{J}+\nabla_{a}\left(\Gamma_{b d}^{d}\right)_{J}\right]+\left(\Gamma_{a b}^{f}\right)_{J}\left(\Gamma_{f d}^{d}\right)_{J}-\left(\Gamma_{a e}^{f}\right)_{J}\left(\Gamma_{f b}^{e}\right)_{J}} \\
{\left[R_{[a b]}\right]_{\mathrm{EGR}}=1 / 2\left[\partial_{a}\left(\Gamma_{b d}^{d}\right)_{J}-\partial_{b}\left(\Gamma_{a d}^{d}\right)_{J}\right]}
\end{gathered}
$$

hence

1.4.3 The EGR curvature scalar

$$
\begin{gathered}
{\left[R_{(a b)}\right]_{\mathrm{EGR}}=R_{a b}-1 / 2\left[\left(g_{a b} \nabla_{d} J^{d}+(1 / 3) J_{a} J_{b}\right]\right.} \\
R_{[a b]}=1 / 6\left(\partial_{a} J_{b}-\partial_{b} J_{a}\right)
\end{gathered}
$$

Applying

$$
\begin{gathered}
R_{\mathrm{EGR}}=g^{d a}\left[R_{d a}\right]_{\mathrm{EGR}}, \\
R_{\mathrm{EGR}}=R-\nabla_{e}\left[g^{d a}\left(\Gamma_{d a}^{e}\right)_{J}\right]-\nabla_{e}\left[g^{d e}\left(\Gamma_{d e}^{e}\right)_{J}\right]-g^{d a}\left[\left(\Gamma_{d a}^{e}\right)_{J}\left(\Gamma_{d e}^{d}\right)_{J}-\left(\Gamma_{d e}^{f}\right)_{J}\left(\Gamma_{f a}^{e}\right)_{J}\right] \\
\text { or } R_{\mathrm{EGR}}=R-1 / 3\left[\nabla_{e} J^{e}+1 / 2 J^{2}\right]
\end{gathered}
$$

\subsection{The EGR field equations}

\subsubsection{The EGR Einstein tensor}

Here the subscript EGR is omitted for clarity purpose.

Unlike the Riemann curvature tensor, the EGR curvature tensor is no longer antisymmetric on indices pair $c d$

$$
R_{c d a b}+R_{d c a b}=2 / 3 g_{c d} J_{a b}
$$

or

$$
R_{a b}^{c d}+R_{a b}^{d c}=2 / 3 g^{c d} J_{a b}
$$

Raising the indice $d$ in (1.32) bis and contracting on $d$ and $b$ as well as on $e$ and $c$, we obtain

$$
R_{a b}^{c d}{ }^{\prime}, d+R_{d a}^{c d}{ }^{\prime},{ }_{c}+R^{c d}{ }_{c d}{ }^{\prime}, a=0
$$

we next replace $R_{c a}^{c d}$ by its value taken from (1.35)bis, and we eventually find

$$
R^{c d}{ }_{c d}^{\prime},{ }_{a}+2 R_{a c}^{c d}{ }^{\prime},{ }_{d}+2 / 3 g^{c d} J_{d a}{ }^{\prime}{ }_{c}=0
$$

that is

$$
\left[R_{(a)}^{(d)}-1 / 2 \delta_{a}^{d} R\right]^{\prime},{ }_{d}=-1 / 3 J_{a}^{d}{ }_{a}^{\prime},{ }_{d}
$$

which is formally the generalized conservation law for the tensor (re-instating the subscript EGR)

$$
\left[G_{d a}\right]_{\mathrm{EGR}}=\left[R_{(d a)}\right]_{\mathrm{EGR}}-1 / 2\left[g_{d a} R_{\mathrm{EGR}}-2 / 3 J_{d a}\right]
$$

We call it the EGR Einstein Tensor. 
In the Riemannian regime, this tensor obviously reduces to the usual Einstein tensor $G_{d a}=R_{d a}-1 / 2 g_{d a} R$ within the classical GR field equations.

\subsubsection{The EGR field equations}

With the equations (1.39) wecan form the EGR source free field equations

$\left[G_{d a}\right]_{\mathrm{EGR}}=0$ which are the tensor transcription of the tensor density equations

$$
\mathfrak{R}^{d a}+\mathscr{B}^{d a}=0
$$

and whose conservation law is expressed by $\left(\Re_{a}{ }^{b}+\mathcal{B}_{a}{ }^{b}\right)^{\prime},{ }_{b}=0$

It is now easy to determine the expression of the tensor $B_{[d a]}$ corresponding to $\mathfrak{B}^{d a}$

$$
B_{[d a]}=-1 / 2\left[g_{d a} 3 / 2 \nabla_{e} J^{e}+1 / 3 J_{d} J_{a}-g_{d a} 1 / 6 J^{2}+2 / 3 J_{d a}\right]
$$

In the strict (ideal) Riemannian regime $\oint^{a}=0$, we would have

$$
\left(\Re_{a}{ }^{b}\right)^{\prime},{ }_{b}=\left(\Re_{a}{ }^{b}\right) ;{ }_{b}=0 \text {, i.e. } \partial_{b} \Re_{a}{ }^{b}-\left\{{ }_{b a}\right\} \Re_{c}{ }^{b}=0
$$

or $\partial_{b} \Re_{a}{ }^{b}-1 / 2 \Re^{c b} \partial_{a} g_{c b}=0$

and eventually $\nabla_{b}\left(R_{a}{ }^{b}-1 / 2 \delta_{a}{ }^{b} R\right)=0$

which is the conserved Einstein 's tensor $G_{a b}$ as inferred from the Bianchi 's identities in classical GR

$$
G_{a b}=R_{a b}-1 / 2 g_{a b} R
$$

When a massive (antisymmetric) tensor $T_{a b}(\rho)$ is present on the right hand side, the field equations become ( $\aleph$ : Einsteins 's constant, with $\mathrm{c}=1)$ :

$$
\left[G_{a b}\right]_{\mathrm{EGR}}=\aleph T_{a b}\left(\rho^{\prime}\right)
$$

In the EGR theory, the mass density $\rho^{\prime}$ is now sligthly modified by its own gravity field.

[ paper to appear in the A.Zelmanov Journal, vol. 3 ].

\section{Relating Spinors with the EGR Theory}

\subsection{General}

The EGR theory exhibits a non vanishing covariant derivative of the fundamental metric tensor.

How, can we relate this non metricity to unify the spin $1 / 2$ field symmetry?

In classical spinor theory, consider the classical $\gamma^{5}$ matrix which includes all other Dirac gamma matrices $\gamma^{a}$.

- In the massless case, the sign of $\gamma^{5}$ distinguishes either one of the anti/Fermionic field.

- Amazingly, for the massive field, the Dirac equation does not require the matrix $\gamma^{5}$ for describing the electron /positon symmetry: one can thus wonder whether the current theory is lacking some consistency.

We may avoid this last ambiguity by achieving a full united theory in the light of our GR extension.

To this effect, we suggest to introduce a 4 x 4 Hermitean matrix that precisely generalizes the Riemannnian gamma matrix $\gamma^{5}$.

This particular matrix is related to the non metric part of the EGR connection.

To prove our conjecture, we develop an EGR formalism based on the essential work by A.Lichnerowicz [Lichnerowicz. A, 1961][Lichnerowicz. A., 1964].

In the massless situation, we will consider the neutrino (Fermionic) particle which is assumed to have zero mass.

For clarity of the text, the mathematics are here kept to a minimum and for deeper analyses, the reader is invited to refer to the suggested bibliography.

\subsection{Riemannian spinor field}

\subsubsection{Space-time orientation}

Let $\Re$ be a $\mathrm{C}^{\infty}$ differentiable 4-manifold which is referred to general frames which are themselves elements of a principal fiber space denoted by $\mathrm{E}(\mathfrak{T})$.

In the Riemannian scheme, this space is denoted by $\mathrm{E}(\mathrm{V} 4)$ and admits a structural group: the homogeneous (or full) Lorentz group denoted by L(4) [Richtmyer. R.D. 1981].

With respect to orthonormal frames $y=\left\{e_{4}, e_{\alpha}\right\}(\alpha=1,2,3)$, the metric can be written on an open neighborhood of the Riemann manifold V4 as 


$$
d s^{2}=g_{a b} \theta^{a} \theta^{b}
$$

where the $\theta^{a}$ are the Pffafian forms in the considered region.

The full (homogeneous) Lorentz group L(4) then includes 4 components:

- The identity component denoted by $\mathbf{L}_{0}(4)$, which is the Lorentz connected group.

- With respect to the considered orthonormal basis, space symmetry provides one element of a second component (including $\left.\mathbf{L}_{0}(4)\right)$ which is denoted by $\mathbf{L}_{1}(4)$ forming the orthochronous group $\left(e_{4} \rightarrow e_{4}, e_{\alpha} \rightarrow-e_{\alpha}\right)$.

- Time symmetry defines a third component (including $\left.\mathbf{L}_{0}(4)\right)$, element of the orthospatial group $\mathbf{L}_{2}(4)\left(e_{4} \rightarrow-e_{4}\right.$, $\left.e_{\alpha} \rightarrow e_{\alpha}\right)$.

- Lastly, the space-time symmetry provides along with $\mathbf{L}_{0}(4)$, an element of the 4th component which defines the Unimodular Lorentz group $\mathbf{L}_{3}(4)\left(e_{4} \rightarrow-e_{4}, e_{\alpha} \rightarrow-e_{\alpha}\right)$.

To the matrix $\mathbf{F}=\left(\mathrm{F}^{b^{\prime}}{ }_{c}\right) \in \mathbf{L}(4)$, can be assigned the relevant component of the Lorentz group in the following way:

The global signature $\varepsilon_{\mathrm{F}}$ of the matrix $\mathbf{F}$ is: $(+/-) 1$ according to the sign of $\operatorname{det} \mathbf{F}$.

The time signature $t_{F}$ of the matrix $\mathbf{F}$ is: $(+/-) 1$ according to the sign of $\mathrm{F}^{4}{ }_{4}$.

The spatial signature $\mathrm{s}_{\mathrm{F}}$ of $\mathbf{F}$ can be obtained by the product $\varepsilon_{\mathrm{F}} \mathrm{t}_{\mathrm{F}}$.

The four components of $\mathbf{L}(4)$ may thus be characterized by the following signatures

$\mathbf{L}_{0}(4): \mathrm{t}_{\mathrm{F}}=1, \mathrm{~s}_{\mathrm{F}}=1 \rightarrow \varepsilon_{\mathrm{F}}=1$

$\mathbf{L}_{1}(4): \mathrm{t}_{\mathrm{F}}=1 \quad, \quad \mathrm{~s}_{\mathrm{F}}=-1 \rightarrow \varepsilon_{\mathrm{F}}=-1$

$\mathbf{L}_{2}(4): \mathrm{t}_{\mathrm{F}}=-1 \quad, \quad \mathrm{~s}_{\mathrm{F}}=1 \rightarrow \varepsilon_{\mathrm{F}}=-1$

$\mathbf{L}_{3}(4): \mathrm{t}_{\mathrm{F}}=-1 \quad, \quad \mathrm{~s}_{\mathrm{F}}=-1 \rightarrow \varepsilon_{\mathrm{F}}=1$

The global orientation of the manifold V4 is then a pseudoscalar denoted by $\varepsilon$, whose

square is 1 , and which is defined with respect to the frames $y$ of $\mathrm{E}(\mathrm{V} 4)$, as the component $\varepsilon_{y}=+/-1$, such that if $y=y^{\prime} \mathbf{F}$, we get $\varepsilon_{y}=\varepsilon_{y}, \varepsilon_{\mathrm{F}}$

As a result, for two global orientations on $\mathrm{V} 4, \varepsilon$ and $\varepsilon$, we have at most two total orientations $\varepsilon$ and $-\varepsilon$.

Using the pfaffian forms $\theta^{a}$, we are now able to define the orientable volume element $\eta$ as

$$
\varepsilon \theta^{4} \Lambda \theta^{1} \Lambda \theta^{2} \Lambda \theta^{3}
$$

The manifold considered here is always understood to be globally hyperbolic.

2.2.2 The Dirac gamma matrices

Let now $\mathbf{R}$ be the real numbers set on which the vector space is defined.

This vector space is spanned by the 16 matrices

$$
\text { I, } \gamma_{a}, \quad \gamma_{a} \gamma_{b}, \quad \gamma_{a} \gamma_{b} \gamma_{c}, \quad \gamma_{a} \gamma_{b} \gamma_{c} \gamma_{d}
$$

where $\mathbf{I}$ is the unit matrix, and the $\gamma^{a}$ are the Dirac matrices. [Dirac. P.A.M., 1931]

(small latin indices $a=b=1,2.3,4$ )

These matrices are formed with the Pauli matrices $\sigma_{k}$

$$
\sigma_{x}=\left(\begin{array}{cc}
0 & 1 \\
1 & 0
\end{array}\right), \quad \sigma_{y}=\left(\begin{array}{cc}
0 & -i \\
i & 0
\end{array}\right), \sigma_{z}=\left(\begin{array}{cc}
1 & 0 \\
0 & -1
\end{array}\right)
$$

which are Hermitean and anticommuting:

$$
\sigma_{i} \sigma_{k}+\sigma_{k} \sigma_{i}=2 \delta_{i k}, \sigma_{i} \sigma_{k}-i \varepsilon_{i j k} \sigma_{j}=\delta_{i k},(i=j=k=1,2,3) .
$$

We recall here further properties of these matrices:

$$
\begin{aligned}
& \gamma_{a} \gamma_{b}+\gamma_{b} \gamma_{a}=2 \eta_{a b} \mathbf{I} \quad\left(\eta_{a b} \text { :Minkowskian tensor }\right) \\
& \left(\gamma^{k}\right)^{2}=-\mathbf{I},\left(\gamma^{4}\right)^{2}=\mathbf{I}, \gamma_{a} \gamma_{b} \gamma_{c} \gamma^{a}=0
\end{aligned}
$$

The matrix $\gamma^{4}$ is Hermitean whereas the $\gamma^{k}$ are non-Hermitean.

The expression $\gamma_{B}^{a A} \partial_{a}$ is known as the Dirac operator and it is customary to omit the spinor indices $A, B$ by simply writing

$$
\gamma_{a}=\gamma_{a B}^{A}
$$


so that we have the usual representation

$$
\gamma^{a} \partial_{a}
$$

Fixing now a metric tensor $g_{a b}$, we write the SR relation (2.2) as

$$
\gamma_{a} \gamma_{b}+\gamma_{b} \gamma_{a}=-2 g_{a b} \mathbf{I}
$$

which is verified in the standard representation of real gamma matrices, with the following elements

\begin{tabular}{|c|c|c|c|c|c|c|c|}
\hline \multirow[t]{4}{*}{$\gamma_{4}$} & $\begin{array}{lllll}0 & 0 & 0 & -1\end{array}$ & $\gamma_{1}$ & $\begin{array}{lllll}0 & 0 & 0 & -1\end{array}$ & $\gamma_{2}$ & 1000 & $\gamma_{3}$ & $00-10$ \\
\hline & $00-10$ & & $\begin{array}{llll}0 & 0 & 1 & 0\end{array}$ & & $\begin{array}{llll}0 & 10 & 0\end{array}$ & & $\begin{array}{lllllllllllll}0 & 0 & -1\end{array}$ \\
\hline & 0100 & & 0100 & & $00-10$ & & -10000 \\
\hline & 1000 & & -1000 & & $\begin{array}{llllll}0 & 0 & 0 & -1\end{array}$ & & $0-100$ \\
\hline
\end{tabular}

(2.7)

We note that

$$
\tilde{\gamma}_{a}=-g^{a a} \gamma_{a}
$$

\subsection{The EGR framework}

\subsubsection{The EGR spinor concept}

From $\mathrm{E}(\mathscr{T})$, we define a principal fiber space denoted by $\mathrm{S}(\mathfrak{T})$ whose each point $z$ represents a general spinor frame: at this stage, it is essential to understand that Spin (4) is not here the structural group.

Tr is therefore a vector space of $4 \times 4$ matrices with complex elements, which is not acted upon by the Spin (4) group.

Let us denote by $\pi$ the canonical projection of $\mathrm{S}(\mathscr{N})$ onto $\Re$, and $\mathbf{p}$ the projection of $\mathrm{S}(\boldsymbol{N})$ onto $\mathrm{E}(\boldsymbol{\Re})$, so that a tensor of $\mathbb{N}$ is referred to its frame by $y=\mathbf{p} z$.

The contravariant 1-spinor $\psi$ at $x \in \mathfrak{N}$, is defined as a mapping $z \rightarrow \psi(z)$ of $\pi^{-1}(x)$ onto $\mathfrak{N}$.

The covariant 1-spinor $\alpha$ at $x$, is a mapping $z \rightarrow \alpha(z)$ of $\pi^{-1}(x)$ onto the space $\mathfrak{\Re}$ dual to $\Re$.

The contravariant 1-spinors $\psi$ form a vector space $\mathrm{S} x$ on the complex numbers, whereas the covariant 1 -spinors $\alpha$ form the vector space $\mathrm{S}^{\prime} x$ dual to $\mathrm{S} x$.

Let $\mathbf{B}_{\mathrm{EGR}}$ be the automorphism into $\mathrm{S} x$ and defined as follows

$$
\mathbf{B}_{\mathrm{EGR}}: \psi \rightarrow\left(\gamma^{5}\right)_{\mathrm{EGR}} \quad \psi
$$

$\mathbf{B}_{\mathrm{EGR}}^{\prime}$ is the relevant automorphism into $\mathrm{S}^{\prime} x$

$$
\mathbf{B}_{\mathrm{EGR}}^{\prime}: \quad \alpha \rightarrow \alpha \quad\left(\gamma^{5}\right)_{\mathrm{EGR}}
$$

We have thus introduced a specific $4 \times 4$ Hermitean matrix denoted by $\left(\gamma^{5}\right)_{\mathrm{EGR}}$ and which generally satisfies

$$
\begin{gathered}
\left(\gamma^{5}\right)_{\mathrm{EGR}} \gamma^{a}+\gamma^{a}\left(\gamma^{5}\right)_{\mathrm{EGR}} \neq 0 \\
{\left[\left(\gamma^{5}\right)_{\mathrm{EGR}}\right]^{2} \neq \mathbf{I}}
\end{gathered}
$$

We now postulate that the gamma matrix $\left(\gamma^{5}\right)_{\mathrm{EGR}}$ is related to the metric EGR covariant derivative $\mathrm{D}_{c} g_{a b} \neq 0$, which in the EGR theory, is linked to the 4-vector $J^{a}$ that spans the non metric part of the EGR global connection $\left(\Gamma_{a b}^{d}\right)_{J}=1 / 6\left(\delta_{a}^{d} J_{b}+\delta_{b}^{d}{ }_{b} J_{a}-3 g_{a b} J^{d}\right)$.

Therefore, we set

$$
\operatorname{tr}\left(\gamma^{5}\right)_{\mathrm{EGR}}=\mathrm{k} J_{b}
$$

where $\mathrm{k}$ is a real positive constant.

This is fully legitimate since the skew components of the Hermitean matrix $\left(\gamma^{5}\right)_{\mathrm{EGR}}$ are always real.

The exact form of the hermitean matrice $\left(\gamma^{5}\right)_{\mathrm{EGR}}$ is of no immediate importance as long as it satisfies the above relations.

\subsection{The EGR Dirac equation}

\subsubsection{The EGR spinor connection}

Within a neighborhood 0 of $\mathrm{E}(\mathscr{\Re})$, we define a connection 1 -form $\Gamma$ that is represented by either of the two 
matrices $\left(\Gamma_{b}^{a}\right)_{\mathrm{EGR}}$ or $\left(\Gamma^{a b}\right)_{\mathrm{EGR}}$ and whose elements are linear forms.

The matrix

$$
(N)_{\mathrm{EGR}}=-1 / 4\left(\Gamma^{a b}\right)_{\mathrm{EGR}} \gamma_{a} \gamma_{b}=-1 / 4\left(\Gamma_{b}^{a}\right)_{\mathrm{EGR}} \gamma_{a} \gamma^{b}
$$

defines the spinor connection corresponding to $\Gamma$.

The elements of $N_{\mathrm{EGR}}$ are given by the local 1-forms

$$
\left(N^{A}{ }_{B}\right)_{\mathrm{EGR}}=-1 / 4\left[\left(\Gamma^{a}{ }_{b}\right)_{\mathrm{EGR}}\right] \gamma_{a}^{A}{ }_{C} \gamma^{b C}{ }_{B}
$$

With the global connection $\left.\Gamma_{c b}^{a}=\left[\left\{{ }_{c b}^{a}\right\}\right\}+\left(\Gamma_{c b}^{a}\right)_{J}\right]$ with respect to the frames in $\mathcal{O}$,

The corresponding coefficients of $\boldsymbol{N}_{\mathrm{EGR}}$ are written

$$
\left(N_{B c}^{A}\right)_{\mathrm{EGR}}=-1 / 4\left[\left(\Gamma_{b c}^{a}\right)_{\mathrm{EGR}}\right] \gamma_{a}^{A}{ }_{C} \gamma^{b C}{ }_{B}
$$

2.4.2 The EGR covariant derivatives of the tensor-spinors

Inspection shows that the EGR absolute differential of the gamma matrices are given by

$$
\mathrm{D} \gamma^{a}=d \gamma^{a}+\left(\Gamma_{b}^{a}\right)_{\mathrm{EGR}} \gamma^{b}+\left[\boldsymbol{N}_{\mathrm{EGR}} \gamma^{a}-\gamma^{a} \boldsymbol{N}_{\mathrm{EGR}}\right]
$$

The EGR covariant differential is still denoted by $\mathrm{D}$.

Let us remark that this differential is generally not zero.

With (2.15) it can also be shown that the EGR covariant derivative of a spinor $\psi^{A}$ is

$$
\mathrm{D}_{c} \psi^{A}=\partial_{c} \psi^{A}+\left(N_{B c}^{A}\right) \mathrm{EGR} \psi^{B}
$$

and for the covariant 1-spinor $\alpha$

$$
\mathrm{D}_{c} \alpha_{A}=\partial_{c} \alpha_{A}-\left(N^{B}{ }_{A c}\right)_{\mathrm{EGR}} \alpha_{B}
$$

By tensor product, one obtains the components of the EGR covariant derivative of any arbitrary tensor-spinor. Introducing now the EGR Dirac operators $W$ and $W^{\prime}$ as

$$
W \psi=\gamma^{a} \mathrm{D}_{a} \psi, \quad W^{\prime} \alpha=-\mathrm{D}_{a} \alpha \gamma^{a}
$$

for a massive spin 1/2 field, the EGR Dirac equations are written

$$
\begin{aligned}
{\left[W-\left(m_{0}\right)^{\prime}\right] \psi } & =0 \\
{\left[W^{\prime}-\left(m_{0}\right)^{\prime}\right] \alpha } & =0
\end{aligned}
$$

where the mass $m_{0}$ is now slightly modified according to the EGR concept.

\subsection{The EGR Lichnerowicz operator}

\subsubsection{Fundamental identities}

For the following demonstrations, we need some important identities, so we first evaluate the EGR vector-spinor $\boldsymbol{T}$ of type ( $\left.{ }_{1}^{1}{ }_{1}\right)$ (contravariant/covariant), omitting the EGR subscript.

$$
T^{a}=R_{b c d}^{a} \gamma^{b} \gamma^{c} \gamma^{d}
$$

Starting from the relation

$$
\left(R_{b c d}^{a}+R_{c d b}^{a}+R_{d b c}^{a}\right) \gamma^{b} \gamma^{c} \gamma^{d}=0
$$

one obtains the identity

$$
T^{a}=R_{b c d}^{a} \gamma^{b} \gamma^{c} \gamma^{d}=2 R_{b}^{a} \gamma^{b}
$$

from which can be inferred

$$
R_{b c d}^{a} \gamma^{c} \gamma^{b} \gamma^{d}=0
$$

finally, we find

$$
R_{a b c d} \gamma^{a} \gamma^{b} \gamma^{c} \gamma^{d}=-2 R_{a b} g^{a b} \mathbf{I}
$$

2.5.2 The EGR curvature tensor-spinor

By perfect analogy with the classical 1-forms theory, we may define the 2-form $\Omega_{\mathrm{S}}$ referred in any bases which are elements of $\mathrm{E}(\mathfrak{T})$, as the EGR spinor connection curvature 2- form

$$
\left[\Omega_{\mathrm{S}}\right]_{\mathrm{EGR}}=d \boldsymbol{N}_{\mathrm{EGR}}+1 / 2\left[\boldsymbol{N}_{\mathrm{EGR}}, \boldsymbol{N}_{\mathrm{EGR}}\right]
$$


Omitting the subscript EGR for $N$, the matrix of $\Omega_{\mathrm{S}}$ is composed of local 2-forms expressed by

$$
\left[\left(\Omega^{A}\right)_{\mathrm{S}}\right]_{\mathrm{EGR}}=d N_{B}^{A}+N^{A}{ }_{C} \Lambda N_{B}^{C}
$$

We call EGR curvature tensor-spinor $\boldsymbol{P}$, related to the EGR spinor connection, the tensor-spinor antisymmetric on $c$ and $h$, whose components in a dual basis, are represented by

$$
\left[\left(\Omega^{A}\right)_{\mathrm{S}}\right]_{\mathrm{EGR}}=1 / 2 P \quad{ }_{B, c h} \theta^{c} \Lambda \theta^{h}
$$

explicitly

$$
P_{B, c h}^{A}=\partial_{c} N_{B h}^{A}-\partial_{h} N_{B c}^{A}+N_{D c}^{A} N_{B h}^{D}-N_{D h}^{A} N_{B c}^{D}-N_{B e}^{A}\left(\Gamma_{h c}^{e}-\Gamma_{c h}^{e}\right)
$$

From the expression of $\boldsymbol{N}_{\mathrm{EGR}}$, we infer

$$
P_{B, c h}^{A}=-1 / 4 \quad\left[R_{b c h}^{a}\right]_{\mathrm{EGR}}\left(\gamma_{a} \gamma^{b}\right)_{B}^{A}
$$

therefore, in accordance with equation (1.03), $\boldsymbol{P}$ verifies the analogue of the Bianchi's identity

$$
\mathrm{D}_{c} P_{B, h e}^{A}+\mathrm{D}_{h} P_{B, e c}^{A}+\mathrm{D}_{e} P_{B, c h}^{A}=0
$$

One can show that the EGR spinor connection curvature is related to the global curvature by

$$
\left[\Omega_{\mathrm{S}}\right]_{\mathrm{EGR}}=-1 / 4\left[\Omega_{b}^{a}\left(\gamma_{a} \gamma^{b}\right)+\Omega\left(\gamma_{a} \gamma^{a}\right)\right]
$$

where $\Omega^{a}{ }_{b}$ is the Riemannian curvature 2-form in the dual basis

$$
\Omega_{b}^{a}=-1 / 2 R_{b c d}^{a} \theta^{c} \Lambda \theta^{d}
$$

and $\Omega$ is the curvature 2-form, contraction of the EGR curvature 2-form:

$$
\Omega_{a}^{a}=-1 / 2\left[R_{a c d}^{a}\right]_{\mathrm{EGR}} \theta^{c} \Lambda \theta^{d}
$$

Let us now denote by

$$
\Delta_{\mathrm{EGR}} \psi=\gamma^{c} \gamma^{d} \quad \mathrm{D}_{c} \mathrm{D}_{d} \psi
$$

the Laplacian acting on the contravariant 1-spinor $\psi$.

This second order operator $\Delta_{\mathrm{EGR}}$ can be explicitly written if we define the Laplacian of a contravariant 1-spinor (resp. covariant) as

$$
\Delta_{\mathrm{EGR}}=W^{2}, \quad\left(\text { resp. } \Delta_{\mathrm{EGR}}=W^{\prime 2}\right)
$$

and expanding

$$
\Delta_{\mathrm{EGR}} \psi=1 / 2\left(\gamma^{c} \gamma^{d}+\gamma^{d} \gamma^{c}\right) \mathrm{D}_{c} \mathrm{D}_{d} \psi+1 / 2 \gamma^{c} \gamma^{d}\left(\mathrm{D}_{c} \mathrm{D}_{d}-\mathrm{D}_{d} \mathrm{D}_{c}\right) \psi
$$

the Ricci identity yields

$$
\left(\mathrm{D}_{c} \mathrm{D}_{d}-\mathrm{D}_{d} \mathrm{D}_{c}\right) \psi^{A}=P_{B, c d}^{A} \psi^{B}
$$

so taking into account (2.7) and (2.27),

$$
\Delta_{\mathrm{EG}} \psi=-\mathrm{D}^{e} \mathrm{D}_{e} \psi-(1 / 8)\left[R_{a b c d}\right]_{\mathrm{EGR}} \gamma^{c} \gamma^{d} \gamma^{a} \gamma^{b} \psi
$$

also due to the symmetry of $P^{A}{ }_{B}, c d$, one finally obtains from (2.24) and (2.32)

$$
W^{2} \psi=\Delta_{\mathrm{EGR}} \psi=-\mathrm{D}^{e} \mathrm{D}_{e} \psi+1 / 4(R)_{\mathrm{EGR}} \psi
$$

also

$$
W^{\prime 2} \alpha=\Delta_{\mathrm{EGR}} \alpha=-\mathrm{D}^{e} \mathrm{D}_{e} \alpha+1 / 4(R)_{\mathrm{EGR}} \alpha
$$

In both cases, we have the same operator

$$
\Delta_{\mathrm{EGR}}=-\mathrm{D}^{e} \mathrm{D}_{e}+1 / 4 R_{\mathrm{EGR}}
$$

with the EGR curvature scalar: $R_{\mathrm{EGR}}=R-1 / 3\left[\nabla_{e} J^{e}+1 / 2 J^{2}\right]$

The operator (2.37) is the EGR version of the Lichnerowicz operator, (or Lichnerowicz-Weitzenböck formula) which is classically used to express the Dirac second order propagation equation.

\section{EGR field degeneracy}

\subsection{A specific gamma matrix}

At the Riemannian approximation, we postulate that $\left(\gamma^{5}\right)_{\mathrm{EGR}} \rightarrow \gamma^{5}$, $\mathfrak{\Re} \rightarrow \mathrm{V} 4$ which is endowed with the time orientation $t$, thus we must find back the classical results of the Riemannian spinor theory.

In this case, at the point $x$ on the manifold $\mathrm{V} 4$, the vector space consisting of all forms and antisymmetric tensors is $\mathbf{S}$ isomorphic to the space of 
all 2-spinors of type (11).

Among all complex valued $\mathrm{p}$-forms $\mu^{(\mathrm{p})}$ one can show that $\exists$ only one form such that

$$
\psi=\mathbf{S} \mu
$$

On V4 endowed with the global orientation $\varepsilon$, is defined the real volume element form (2.1), to which corresponds by $\mathbf{S}$ the (11) spinor $\mathbf{S} \eta$.

We now define the matrix isomorphic to $\eta$ as

$$
\gamma^{5}=i \mathbf{S} \eta
$$

and displaying the components, we can show that

$$
\gamma^{5}=(i / 4 !) \gamma^{a} \gamma^{b} \gamma^{c} \gamma^{d} \eta_{a b c d}
$$

On an open neighbourhood of V4 we may express this matrix with respect to spinorial indices as

$$
\gamma^{5}=i \varepsilon \gamma^{4} \gamma^{1} \gamma^{2} \gamma^{3}
$$

Since we have adopted the standard representation for the gamma matrices, this spinor is purely imaginary and satisfies

$$
\begin{gathered}
\tilde{\gamma}^{5}=\gamma^{5} \\
\gamma^{5} \gamma^{a}+\gamma^{a} \gamma^{5}=0 \\
\left(\gamma^{5}\right)^{2}=\mathbf{I}
\end{gathered}
$$

These relations clearly appear as a particular Riemannian case of the EGR relations (2.11) and (2.12).

The contravariant 1 -spinor $\psi$, has also now its covariant 1 -spinor counterpart which we write as

$$
{ }^{\circ} \psi=\mathrm{t} \tilde{\psi} \beta
$$

and which is classically known as the Dirac conjugate. (see next § 3.2)

Conversely, any covariant 1 -spinor $\alpha$ is now the image of the contravariant 1 -spinor $\mathrm{t} \beta \alpha$.

Reducing to the Riemannian scheme leads to

$$
J^{a}=\mathrm{k} \operatorname{tr}\left(\gamma^{5}\right)_{\mathrm{EGR}}=0,
$$

which thereby re-instates the metricity of the classical spinor theory for which holds the known condition

$$
\operatorname{tr} \gamma^{5}=0
$$

\subsection{The Charge conjugation and the Adjoint operation}

There exists an antilinear mapping $\mathbf{C}$ of $\mathbf{S} x$ onto itself, which maps a 1-spinor $\psi$ to another 1-spinor such as

$$
\mathbf{C}: \psi \rightarrow \psi \mathbf{C}=\psi^{*}
$$

We readily see that $\mathbf{C}^{2}=$ Identity. $(\psi \rightarrow \psi)$

$\mathbf{C}$ is known as the charge conjugate operation.

In particular, the charge conjugate of the covariant 1 -spinor $\alpha$ is

$$
\begin{gathered}
\mathbf{C} \alpha=\alpha^{*}, \text { hence } \\
(\mathbf{C} \alpha, \psi)=(\alpha, \mathbf{C} \psi)^{*}
\end{gathered}
$$

The relation (2.6) results from the identity $\left(u^{a} \gamma_{a)^{2}}=-\left(u^{a} u^{b} g_{a b}\right) \mathbf{I}\right.$ where $u_{a} \in \mathcal{C}$ (complex numbers), thus from (2.8) we find

$$
\gamma_{4} \gamma_{a} \gamma_{4}{ }^{-1}=-\tilde{\gamma}_{a}
$$

Introducing now the Hermitean matrix

which verifies

\begin{tabular}{|l|cccc|}
\multicolumn{3}{c}{$\beta$} & $=$ & $i \gamma_{4}$ \\
\hline$\beta$ & 0 & 0 & 0 & $-i$ \\
& 0 & 0 & $-i$ & 0 \\
& 0 & $i$ & 0 & 0 \\
& $i$ & 0 & 0 & 0 \\
\hline
\end{tabular}




$$
\beta^{2}=\mathbf{I}
$$

we derive from (3.9) the important relation

$$
\beta \gamma_{a} \beta^{-1}=-\tilde{\gamma}_{a}
$$

By defining an antilinear mapping $\mathbf{A}$ of $\mathrm{S} x$ onto $\mathrm{S}$ ' $x$, as

$$
\text { A : } \quad \psi \rightarrow{ }^{o} \psi=\mathrm{t} \tilde{\psi} \beta
$$

we have the Dirac adjoint operation $\mathbf{A}$.

\subsection{The fundamental spinor 2-form}

We now consider a contravariant 1-spinor $\psi$ which satisfies $\mathbf{C} \psi=\psi^{*}$

thus AC $\psi=\mathrm{t} \psi^{\mathrm{T}} \boldsymbol{\beta}$

On the other hand $\mathbf{A} \psi=\mathrm{t} \tilde{\psi} \beta$, so we infer

$$
\mathbf{C A} \Psi=\mathrm{t} \psi^{\mathrm{T}} \beta^{*} \text { i.e. } \mathbf{C A} \psi=-\mathrm{t} \psi^{\mathrm{T}} \beta
$$

Therefore $\mathbf{A C} \psi=-\mathbf{C A} \psi$

The Dirac adjoint operator and the charge conjugation anticommute on the 1-spinors.

We define now a covariant spinor 2-form denoted by $\omega$, which allows to identify either the contravariant or covariant 1-spinor.

This form can be regarded as the analogue of the fundamental tensor $\boldsymbol{g}$ defined on the riemannian manifold V4.

Consider again the contravariant 1-spinor $\psi$ to which corresponds the covariant 1-spinor $\mathbf{A C} \psi$.

$\omega$ then represents an isomorphism (linear mapping of $\mathrm{S} x$ onto $\mathrm{S} x$ ), that is a covariant 2-spinor whose components are $\omega_{A B}$, so that

$$
(\omega \psi)_{A}=\omega_{A B} \psi^{B}
$$

and which is antisymmetric.

A fundamental vector-spinor $\gamma$ of type $\left({ }_{1}^{1}\right)$ can be contracted by applying $\omega$ to yield a covariant vector 2-spinor which is symmetric on spinor indices

$$
\gamma_{a A B}=\omega_{A C} \gamma_{a B}^{C}=\gamma_{a B A}
$$

Unlike the EGR picture (2.16), the Riemannian covariant differential of the gamma matrices is known

to be zero

$$
\nabla \gamma^{a}=d \gamma^{a}+\Gamma_{b}^{a} \gamma^{b}+N \gamma^{a}-\gamma^{a} \boldsymbol{N}=0
$$

Let us now summarize the results obtained above

a) In the canonical spinor connection, the absolute differential of the fundamental tensor-spinor $\gamma$ is zero.

b) The charge conjugation and the Dirac adjoint operation commute with the covariant derivative.

c) The covariant derivative of the spinor 2-form $\omega$ is zero.

In the EGR theory, it is obvious that none of these conditions are fulfilled, in particular the condition $c$ ) which like in Riemannian GR, reflects here the metricity condition of the classical spinor theory.

\subsubsection{The double spinor formulation}

At the Riemannian approximation, $\mathbf{B}_{\mathrm{EGR}} \rightarrow \mathbf{B}$, and now verifies $\mathbf{B}^{2}=$ Identity, $(\psi \rightarrow \psi)$.

Therefore, B admits two eigenvalues: +/- 1, so we may decompose $\mathrm{S} x$ into a sum $(\mathrm{S} x)_{+} \otimes(\mathrm{S} x)_{-}$of the eigen subspaces of $\mathbf{B}$.

An element of $(\mathrm{S} x)_{+}$is a spinor of positive type denoted by $\psi_{+}$

An element of $(\mathrm{S} x)$. is a spinor of negative type denoted by $\psi_{\text {. . }}$

We check that

$$
\mathbf{B} \psi_{+}=\psi_{+}
$$

we may apply the charge conjugation to $\mathbf{B} \psi$

$$
\mathbf{C B} \psi=\gamma^{5} \psi^{*}=-\gamma^{5} \psi^{*}=\gamma^{5} \psi \mathbf{C}=-\mathbf{B C}
$$


hence

$$
\mathbf{C B}=-\mathbf{B C} \text {, and } \mathbf{C B}^{\prime}=-\mathbf{B}^{\prime} \mathbf{C}
$$

Applying now the Dirac adjoint operation $\mathbf{A}$ to $\mathbf{B} \psi$, we have

$$
\mathbf{A B} \psi=\mathrm{t} \psi \tilde{\psi} \tilde{\gamma}^{5} \beta=\mathrm{t} \tilde{\psi} \gamma^{5} \beta=-\mathrm{t} \tilde{\psi} \beta \gamma^{5}=-{ }^{\circ} \psi \gamma^{5}=\mathbf{B}^{\mathbf{A}} \mathbf{A} \psi
$$

thus

$$
\mathbf{A B}=-\mathbf{B}^{\prime} \mathbf{A}, \quad \mathbf{A B}^{\prime}=-\mathbf{B} \mathbf{A}
$$

Under charge conjugation the following relation holds $\quad \mathbf{C B} \psi_{+}=\mathbf{C} \psi_{+}$

which according to (3.19) yields $\mathbf{B}\left(\mathbf{C} \psi_{+}\right)=-\mathbf{C} \psi_{+}$

C maps ( $\mathrm{S} x)_{\text {- onto }}(\mathrm{S} x)_{+}$and vice versa.

Furthermore, if $\psi \in \mathrm{S} x$, we have

$$
\psi=\psi_{+}+\psi
$$

from which is inferred $\mathbf{B} \alpha=\psi_{+}-\psi_{\text {- }}$, hence

$$
\begin{gathered}
\psi_{+}=1 / 2(\mathbf{I}+\mathbf{B}) \psi_{,} \quad \psi_{-}=1 / 2(\mathbf{I}-\mathbf{B}) \psi \\
{ }^{\circ} \psi_{+}=1 / 2\left(\mathbf{I}-\mathbf{B}^{\prime}\right){ }^{\circ} \psi,{ }^{\circ} \psi \quad=1 / 2\left(\mathbf{I}+\mathbf{B}^{\prime}\right)^{\circ} \psi
\end{gathered}
$$

3.3.2 Riemannian Dirac operations

Let us now express the Dirac operators as

$$
Z \psi=\gamma^{a} \nabla_{a} \psi, Z^{\prime} \alpha=-\nabla_{a} \alpha \gamma^{a}
$$

The charge conjugation $\mathbf{C}$ commutes with the covariant derivative and therefore

$$
\mathbf{C} Z \psi=Z \mathbf{C} \psi, \quad \mathbf{C} Z^{\prime} \alpha=Z^{\prime} \quad \mathbf{C} \alpha
$$

The Dirac adjoint operator $\mathbf{A}$ also commutes with the covariant derivative and we can show that

$$
\mathbf{A} Z \psi=Z^{\prime} \mathbf{A} \psi, \quad \mathbf{A} Z^{\prime} \quad \alpha=Z \mathbf{A} \alpha
$$

From these two relations, we infer

$$
\omega Z^{\prime} \psi=Z^{\prime} \omega \psi
$$

3.3.3 The Dirac current vector density

The Dirac Lagrangian for a massive fermion field is shown to be

$$
\mathfrak{L}_{D}=1 / 2\left[{ }^{\circ} \psi \gamma^{a} \nabla_{a} \psi-\left(\nabla_{a}{ }^{\circ} \psi\right) \gamma^{a} \psi\right]-m_{0}{ }^{\circ} \psi \psi
$$

An alternative expression is given by

$$
\mathfrak{L}_{D}={ }^{\circ} \psi\left[\gamma^{a} \nabla_{a}-m_{0}\right] \psi
$$

Since these forms differ only by a divergence which vanishes at infinity, they generate the same action and correspond to the same physics.

Following Noether 's theorem, we now apply the invariance rule to $\mathfrak{L}_{D}(3.26)$ upon the global transformations (where $\mathrm{U}$ is a positive scalar).

$$
\psi \rightarrow e^{i \mathrm{U}} \psi,{ }^{\circ} \psi \rightarrow{ }^{\circ} \psi e^{-i \mathrm{U}}
$$

for linear transformations of $\psi$, the corresponding Lagrangian variation is

$$
\delta \mathfrak{L}_{D}=i^{\circ} \psi \gamma^{a} \psi \nabla_{a} \delta \mathrm{U}=\nabla_{a}\left(i^{\circ} \psi \gamma^{a}{ }^{\circ} \psi \delta \mathrm{U}\right)-\nabla_{a}\left(i^{\circ} \psi \gamma^{a} \psi\right) \delta \mathrm{U}
$$

from which we expect to infer a current density $\left(j_{a}\right)_{D}$ through a classical action variation

$$
\delta \varsigma_{D}=-\int \nabla_{a}\left(j^{a}\right)_{D} \delta \mathrm{U} \eta=0
$$

where we have set

$$
\left(j^{a)}{ }_{D}=i^{\circ} \psi \gamma^{a} \psi\right.
$$

If $\psi$ is a solution of the field equations $\delta \varsigma_{D}$ vanishes for any $\delta \mathrm{U}$, so

$$
\nabla^{a}\left(j_{a}\right)_{D}=0
$$

Thus we have defined the Dirac current vector density $\left(j^{a}\right)_{D}$ which is a true vector.

To prove this, we write $\left(j^{a}\right)_{D}$ with the aid of (3.12) 


$$
\left(j^{a}\right)_{D}=i \mathrm{t} \tilde{\psi} \beta \gamma^{a} \psi
$$

by applying the usual adjoint operation $\left(j^{a}\right)_{D}{ }^{*}=-i \mathrm{t} \tilde{\psi} \tilde{\gamma} \tilde{\gamma}^{a} \beta \psi$ and taking into account $\tilde{\gamma}^{a} \beta=-\beta \gamma^{a}$, we eventually find

$$
\left(j^{a}\right)_{D}^{*}=i \mathrm{t} \tilde{\psi} \gamma^{a} \psi=i^{\circ} \psi \gamma^{a} \psi=\left(j^{a}\right)_{D}
$$

which concludes the demonstration.

3.3.4 Special features of the Dirac current

In the massless case $m_{0}=0$, the Dirac current density can be split up in 2 parts.

Let us write the first part as

$$
\begin{gathered}
{\left[\left(j^{a}\right)_{D}\right]_{\mathrm{S}}=-\left(i / 4 m_{0}\right) \nabla_{b}\left[{ }^{\circ} \psi\left(\gamma^{b} \gamma^{a}-\gamma^{a} \gamma^{b}\right)\right] \psi} \\
=-\left(i / 4 m_{0}\right)\left[\left(\nabla_{b}{ }^{\circ} \psi\right)\left(\gamma^{b} \gamma^{a}-\gamma^{a} \gamma^{b}\right) \psi+{ }^{\circ} \psi\left(\gamma^{b} \gamma^{a}-\gamma^{a} \gamma^{b}\right) \nabla_{b} \psi\right]
\end{gathered}
$$

From the relation (2.6), we find

$$
\left[\left(j^{a}\right)_{D}\right]_{\mathrm{S}}=-\left(i / 2 m_{0}\right)\left[\nabla_{b} \circ \psi\left(\gamma^{b} \gamma^{a}+g^{a b} \mathbf{I}\right) \psi+{ }^{\circ} \psi\left(-g^{a b} \mathbf{I}-\gamma^{a} \gamma^{b}\right) \nabla_{b} \psi\right]
$$

that is

$$
\left[\left(j^{a}\right)_{D}\right]_{\mathrm{S}}=-\left(i / 2 m_{0}\right)\left[Z^{\prime}{ }^{\circ} \psi \gamma^{a} \psi+{ }^{\circ} \psi \gamma^{a} Z \psi+{ }^{\circ} \psi \nabla^{a} \psi-\left(\nabla^{a}{ }^{\circ} \psi\right) \psi\right]
$$

By virtue of the Dirac equations $Z \psi=m_{0} \psi$ and $Z^{1 \circ} \psi=m_{0}{ }^{\circ} \psi$, we eventually get

$$
\left[\left(j^{a}\right)_{D}\right]_{\mathrm{S}}=i^{\circ} \psi \gamma^{a} \psi+\left(i / 2 m_{0}\right)\left[{ }^{\circ} \psi \nabla^{a} \psi-\left(\nabla^{a^{\circ}} \psi\right) \psi\right]
$$

The Dirac current density $j^{a}$ can therefore be decomposed as

$$
\left(j^{a}\right)_{D}=i^{\circ} \psi \gamma^{a} \psi=\left[\left(j^{a}\right)_{D}\right]_{\mathrm{S}}+\left[\left(j^{a}\right)_{D}\right]_{\mathrm{OR}}
$$

with the (field) spin current

$$
\left[\left(j^{a}\right)_{D}\right]_{\mathrm{S}}=-\left(i / 4 m_{0}\right) \nabla_{b}\left[{ }^{\circ} \psi\left(\gamma^{b} \gamma^{a}-\gamma^{a} \gamma^{b}\right) \psi\right]
$$

and the (field) orbital current

$$
\left[\left(j^{a}\right)_{D}\right]_{\mathrm{OR}}=-\left(i / 2 m_{0}\right)\left[{ }^{\circ} \psi \nabla^{a} \psi-\left(\nabla^{a}{ }^{\circ} \psi\right) \psi\right]
$$

A similar result can be found in [Bogoliubov. N.N., 1959].

\subsection{The Riemann Dirac equation}

\subsubsection{The free field Dirac equation}

Consider the contravariant 1-spinor $\psi$. Since the covariant derivative of the matrix $\gamma^{5}$ is zero, we have

$$
\text { B } Z \psi=\gamma^{5} \gamma^{a} \nabla_{a} \psi=-\gamma^{a} \gamma^{5} \nabla_{a} \psi=-\gamma^{a} \nabla_{a}\left(\gamma^{5} \psi\right)=-Z \mathbf{B} \psi
$$

hence

$$
\mathbf{B} Z=-Z \mathbf{B}, \mathbf{B}^{\prime} \quad Z^{\prime}=-Z^{\prime} \mathbf{B}^{\prime}
$$

The Dirac operators act on the type of positive (resp.negative) spinors, whereas the spinor Laplacian $\Delta$ preserves either type.

The Dirac massive spin $1 / 2$ field may be described by a contravariant 1 -spinor $\psi$ that satisfies the free field equation (no external interacting field).

and the Dirac adjoint ${ }^{\circ} \psi$ satisfies

$$
\left(Z-m_{0}\right) \psi \quad=0
$$

$$
\left(Z^{\prime}-m_{0}\right)^{\circ} \psi=0
$$

In a more conventional manner, the Dirac equation for a free particule with rest mass $m_{0}$, reads

$$
\left[\gamma^{a} \nabla_{a}-m_{0}\right] \psi=0
$$

3.4.2 Massive Dirac field interacting with an electromagnetic field

Let us consider here the lagrangian for a charged Dirac massive field coupled with a potential $A_{a}$

$$
\mathfrak{L}\left(\psi, A_{a}\right)=\mathfrak{L}(\psi)+\mathfrak{L}\left(A_{a}\right)-\mathrm{e} \psi A_{a}\left(j^{a}\right)_{D}
$$

the coupling constant $\mathrm{e}$ is here taken as a negative charge. (e.g. electron)

Taking into acccount the expression of the Dirac current density 


$$
\left(j^{a}\right)_{D}=i\left({ }^{\circ} \psi \gamma^{a} \psi\right)
$$

we shall evaluate the variation of the lagrangian $\mathfrak{L}\left(\psi, A_{a}\right)$.

After a simple but lengthy calculation, we obtain (omitting ${ }_{D}$ in $j$ )

$$
\begin{aligned}
\delta \mathfrak{L}\left(\psi, A_{a}\right) & =\left[\delta^{\circ} \psi\left(\gamma^{a}\left(\nabla_{a}-i \mathrm{e} A_{a}\right) \psi-m_{0} \psi\right)\right]+\left[\left(-\left(\nabla_{a}+i \mathrm{e} A_{a}\right)^{\circ} \psi \gamma^{a}-m_{0} \psi\right) \delta \psi\right] \\
& +\left[-\nabla^{b}\left(\nabla_{b} A_{a}-\nabla_{a} A_{b}\right)-\mathrm{e} j_{a}\right] \delta A^{a}+\text { divergence term. }
\end{aligned}
$$

Extremizing the action defined from $\mathfrak{L}\left(\psi, A_{a}\right)$, we get two field equations

$$
\begin{aligned}
& \gamma^{a}\left(\nabla_{a}-i \mathrm{e} A_{a}\right) \psi=m_{0} \psi \\
& -\left(\nabla_{a}+i \mathrm{e} A_{a}\right)^{\circ} \psi \gamma^{a}=m_{0}{ }^{\circ} \psi
\end{aligned}
$$

and

$$
\delta d \boldsymbol{A}=\mathrm{e} \boldsymbol{j}
$$

Under the $\mathbf{C}$ operation, the following transformations take place

$$
\psi \rightarrow \psi^{\mathrm{C}}, j \rightarrow-j
$$

Applying the Dirac adjoint operation $\mathbf{A}$, the first equation becomes

$$
\gamma^{a}\left(\nabla_{a}+i \mathrm{e} A_{a}\right) \psi^{\mathrm{C}}=m_{0} \psi^{\mathrm{C}}
$$

which is interpreted as the positon equation wherein the anti-electron appears when $\psi^{\mathrm{C}}$ is substituted to $\psi$.

\subsection{The spin canonical energy-momentum tensor}

\subsubsection{The massive field}

Let $\mathrm{L}_{\xi}$ be the Lie derivative operation with respect to an arbitrary vector field $\xi$.

Our aim here is to determine the expression of an energy momentum tensor which follows from the general varied action according to the procedure detailed in [Landau. L., 1962] and where

$$
\delta \varsigma=\int \xi^{b}\left(T^{a}{ }_{b}\right) ;{ }_{a}(-g)^{1 / 2} d^{4} x=0
$$

We start by evaluating the Lie derivative of the lagrangian $\mathfrak{L}_{D}$

$$
\mathrm{L}_{\xi} \mathfrak{L}_{D}=\xi^{a} \nabla_{a} \mathfrak{L}_{D}
$$

Using in particular the identities

$$
\begin{gathered}
\gamma^{b}\left(\nabla_{a} \nabla_{b}-\nabla_{b} \nabla_{a}\right) \psi=-1 / 4 R_{a b c d} \gamma^{b} \gamma^{c} \gamma^{d} \psi=-1 / 2 R_{a b} \gamma^{b} \psi \\
\left(\nabla_{a} \nabla_{b}-\nabla_{b} \nabla_{a}\right){ }^{\circ} \psi \gamma^{b}=1 / 4 R_{a b c d}{ }^{\circ} \psi \gamma^{c} \gamma^{d} \gamma^{b}=-1 / 2 R_{a b}{ }^{\circ} \psi \gamma^{b}
\end{gathered}
$$

we eventually find after a ferocious algebra

$$
\mathrm{L}_{\xi} \mathfrak{L}_{D}=1 / 2 \xi^{a} \nabla_{b}\left[\left(\nabla_{a} \psi\right) \gamma^{b} \psi-\gamma^{b} \nabla_{a} \psi\right]+\xi^{a}\left[{ }^{\circ} \psi \nabla_{a}\left(Z-m_{0}\right) \psi+\nabla_{a}\left(Z^{\prime}-m_{0}\right) \psi^{\circ} \psi\right]
$$

The last two terms cancel out for the Dirac solutions.

On the other hand,

$$
\mathrm{L}_{\xi} \mathfrak{L}_{D}=\xi^{a} \nabla_{a} \mathfrak{L}_{D}=\xi^{a} \nabla_{b}\left[\delta^{b}{ }_{a} \mathfrak{L}_{D}\right]
$$

hence, applying (3.48) the equations (3.50) reduce to a conservation law

$$
\nabla_{b}\left[1 / 2\left(\left(\nabla_{a} \psi\right) \gamma^{b} \psi-{ }^{\circ} \psi \quad \gamma^{b} \nabla_{a} \psi\right)-\delta^{b}{ }_{a} \mathfrak{L}_{D}\right]=0
$$

which holds for the mixed indices energy-momentum tensor

$$
T_{a}^{b}=\left[1 / 2\left(\left(\nabla_{a}{ }^{\circ} \psi\right) \gamma^{b} \psi-{ }^{\circ} \psi \quad \gamma^{b} \nabla_{a} \psi\right)-\delta^{b}{ }_{a} \mathfrak{L}_{D}\right]
$$

With the Dirac solutions (3.38) and (3.39), inspection shows that the term $\delta^{a}{ }_{b} \mathfrak{L}_{D}$ vanishes, so the spin canonical energy-momentum tensor can be finally written

$$
T_{a b}=1 / 2\left[\left(\nabla_{a}{ }^{o} \psi\right) \gamma_{b} \psi-{ }^{\circ} \psi \quad \gamma_{b}\left(\nabla_{a} \psi\right)\right]
$$

This tensor is still antisymmetric which agreeably copes with the framework of the EGR theory The spin canonical energy momentum tensor (which is shown to be real), may be symmetrized by adding the divergence of another antisymmetric tensor

$$
\left(T^{a b}\right)_{\text {sym. }}=T^{a b}+1 / 2 \nabla_{c} S^{c b a}
$$

$S^{c b a}=1 / 4{ }^{\circ} \psi\left(\sigma^{c b} \gamma^{a}+\gamma^{a} \sigma^{c b}\right) \psi$ is called the moment spin tensor. 
It should not be confused with the spin tensor $\sigma^{a b}=1 / 2\left(\gamma^{a} \gamma^{b}-\gamma^{b} \gamma^{a}\right)$

This type of symmetrization procedure can also be found in [8] which results in the same conserved law

$$
\nabla_{b}\left(T_{a}^{b}\right)_{\text {sym. }}=0
$$

3.5.2 The massless field

We write the Lagrangian $\mathfrak{L}_{+}$for $\psi_{+}$as before (omitting the subscript ${ }_{D}$ )

$$
\mathfrak{L}_{+}=1 / 2\left[\left({ }^{\circ} \psi_{+}\right) \gamma^{a} \nabla_{a} \psi_{+}-\nabla_{a}\left({ }^{\circ} \psi_{+}\right) \gamma^{a} \psi_{+}\right]
$$

It can be expressed with the spinor $\gamma^{5}$ by taking into account (2.46) and (2.47)

$$
\mathfrak{L}_{+}=1 / 8\left[{ }^{0} \psi \quad\left(\mathbf{I}-\gamma^{5}\right) \gamma^{a}\left(\mathbf{I}+\gamma^{5}\right) \nabla_{a} \psi-\nabla_{a}{ }^{\circ} \psi \quad\left(\mathbf{I}-\gamma^{5}\right) \gamma^{a}\left(\mathbf{I}+\gamma^{5}\right) \psi\right]
$$

we then have $\left(\mathbf{I}-\gamma^{5}\right) \gamma^{a}\left(\mathbf{I}+\gamma^{5}\right)=2 \gamma^{a}+2 \gamma^{a} \gamma^{5}$, and the lagrangian is finally

$$
\mathfrak{L}_{+}=1 / 4\left[{ }^{\circ} \psi \gamma^{a} \nabla_{a} \psi-\left(\nabla_{a}^{\circ} \psi\right) \gamma^{a} \psi\right]+1 / 4\left[{ }^{\circ} \psi \gamma^{a} \gamma^{5} \nabla_{a} \psi-\left(\nabla_{a}{ }^{\circ} \psi\right) \gamma^{a} \gamma^{5} \psi\right]
$$

From the Lagrangian (3.56) can be derived the Riemannian spin canonical energy-momentum tensor for $\psi$ :

$$
\begin{gathered}
\left(T^{a b}{ }^{a}\right)(\psi)=1 / 2\left[\left(\nabla^{a}{ }^{\circ} \psi\right) \gamma^{b} \psi-\gamma^{b} \nabla^{a} \psi\right]+1 / 2\left[\left(\nabla^{a}{ }^{\circ} \psi\right) \gamma^{b} \gamma^{5} \psi+{ }^{\circ} \psi \gamma^{5} \gamma^{b} \nabla^{a} \psi\right] \text {, i.e. } \\
\left(T^{a b}{ }_{+}\right)(\psi)=1 / 2 T^{a b}(\psi)+M^{a b}(\psi)
\end{gathered}
$$

with

$$
M^{a b}(\psi)=1 / 2\left[\left(\begin{array}{lll}
\nabla^{a} & { }^{\circ} \psi
\end{array}\right) \gamma^{b} \gamma^{5} \psi \quad+{ }^{\circ} \psi \quad \gamma^{5} \gamma^{b} \nabla^{a} \psi\right]
$$

Replacing $\psi$ by its charge conjugate $\psi^{\mathrm{C}}$, the canonical tensor $\left(T^{a b}{ }_{+}\right)(\psi)$ becomes

$$
\left(T^{a b}{ }_{+}\right)\left(\psi^{\mathrm{C}}\right)=1 / 2\left[T^{a b}(\psi)-M^{a b}(\psi)\right]
$$

This tensor describes an anti-spin $1 / 2$ field state which is determined by the sign of the matrix $\gamma^{5}$.

In the same way, we would find for $\left(\psi_{-}\right)$, the Riemannian symmetry

$$
\begin{aligned}
\left(T^{a b}\right)(\psi) & =1 / 2\left[T^{a b}(\psi)-M^{a b}(\psi)\right] \\
\left(T^{a b}\right)\left(\psi^{\mathrm{C}}\right) & =1 / 2\left[T^{a b}(\psi)+M^{a b}(\psi)\right]
\end{aligned}
$$

Hence, we are in the presence of 4 fields associated with 4 distinct types of particles

which can be interpreted as follows

$$
\begin{aligned}
& \psi_{+}: v, \mathbf{C} \psi_{+}=\left(\psi_{-}\right)^{\mathrm{C}}:{ }^{\circ} v \\
& \psi_{-}:{ }^{\circ} v^{\prime}, \mathbf{C} \psi_{-}=\left(\psi_{+}\right)^{\mathrm{C}}: v^{\prime}
\end{aligned}
$$

$v$ : electron neutrino. $\quad v^{\prime}:$ meson neutrino.

${ }^{\circ} v$ : electron antineutrino. $\quad{ }^{\circ} v '$ ': meson antineutrino.

The neutrinic fields described here are evidently assumed to be massless.

\section{Conclusions and outlook}

The EGR theory briefly sketched in section 1, has enabled us to introduce the general Hermitean $4 \times 4$ matrix $(\gamma$ $\left.{ }^{5}\right)_{\mathrm{EGR}}$ which reduces to the usual $\gamma^{5}$ matrix when the Riemannian approximation is considered.

The choice for the generalized matrix $\left(\gamma^{5}\right)_{\mathrm{EGR}}$ is strongly suggested by the fact that the Riemannian matrix $\gamma^{5}$ does not appear in the massive spin $1 / 2$ (anti) field equation.

By generalizing $\gamma^{5}$ in the framework of he EGR theory, we are able to obtain a unified theory that potentially contains the Fermion-anti Fermion field symmetry revealed in the Riemannian geometry. Before concluding, let us stress one more important point.

The above results displaying the Riemannian (anti) Fermionic field states may only be inferred from the antisymmetric form of the spin canonical energy-momentum tensor $T^{a b}$.

Using the corresponding symmetrized tensor $\left(T^{a b}\right)_{\text {sym. }}$ does not permit to exhibit the splittings that lead to these fields.

This would lend support to the fact that the genuine field theory is intrinsically antisymmetric in strict accordance with the EGR theory. Eventually, this paper deals with spin $1 / 2$ fields only.

Our united concept deserves further scrutiny as for example the analysis of the Rarita-Schwingerspin 3/2 field 
(so-called gravitinos ' field).

In such a perspective, it would fully agree with the theory suggested by Louis de Broglie postulating the existence of a hidden thermostat [De Broglie. L, 1968] [Vigier. J.P., 1954], which we explicited and generalized in the strict Riemannian geometry [Marquet.P., 2009]-[ Marquet.P., 2010].

\section{References}

Bogoliubov. N.N., Shirkov. D.V. (1959). Introduction of the Theory of Quantized Field. Interscience. New-York.

De Broglie. L. (1968). Thermodynamique Relativiste et Mécanique Ondulatoire. Ann. Institut Henri Poincaré, vol. IX, no. 2, 89-108.

Dirac. P.A.M. (1931). The Principles of Quantum Mechanics. PUF. Paris.

Greiner. W., Reinhardt .J. (1996). Field quantization. p.121-122. Springer. Berlin.

Landau. L., E.Lifshitz .E. (1962). The Classical Theory of Fields. Addison-Wesley, Reading, Massachusetts.

Lichnerowicz. A. (1961). Propagateurs et Commutateurs en Relativité Générale. Institut des Hautes Etudes Scientifiques. Paris. Publications mathématiques no. 10.

Lichnerowicz. A. (1964). Champ de Dirac, Champ du neutrino et transformations C, P,T sur un espace courbe. Ann. Institut Henri Poincaré, sec. A: vol. 3, p. 233-290. Paris.

Marquet. P. (2009). The EGR Theory: an extended formulation of GR. The Abraham Zelmanov Journal, vol. 2.

Marquet.P. (2009). On the Physical Nature of the Wave Function: A new Approach through the EGR Theory. The Abraham Zelmanov Journal, vol. 2.

Marquet.P. (2010). The Matter-Anti Matter Concept revisited. Progress in Physics, vol. 2.

Richtmyer. R.D. (1981). Principles of Advanced Mathematical Physics (vol.II). Springer. Berlin.

Vigier. J.P., Bohm. D. (1954). Model of the causal interpretation of Quantum theory in terms of a fluid with irregular fluctuations. Physical Review, vol.96, no.1, 208-216. 